Tung-Ping Su

Departamento de Psiquiatría,

Hospital General de Veteranos-Taipei, $N^{o} 201$, Sec. 2, Shih-Pai Road, Taipei 112, Taiwán, ROC División de Psiquiatría, Facultad de Medicina, Universidad Nacional Yang-Ming, Taipei, Taiwán, ROC Correo electrónico: tpsu@vghtpe.gov.tw (T.-P.Su)

Ian-Kai Shan Kelly Chang

Departamento de Psiquiatría, Hospital General de Veteranos-Taipei, $N^{\circ} 201$, Sec. 2, Shih-Pai Road, Taipei 112, Taiwán, ROC Instituto de Medicina Clínica,

I.-Hua Wei Departamento de Anatomía y Biología Celular, Facultad de Medicina, Universidad Nacional de Taiwán, Taipei, Taiwán, ROC

\section{Trastorno obsesivo-compulsivo y tratamiento con clozapina en 200 pacientes con esquizofrenia de comienzo reciente o trastornos relacionados}

Señor:

La clozapina se ha asociado con la presencia de sintomas obsesivo-compulsivos en los pacientes con esquizofrenia $[2,3,6,7]$. Sin embargo, esta asociación no se encuentra de manera inequívoca [5].

Identificamos trastorno obsesivo-compulsivo (TOC) según los criterios del DSM-IV antes y después de la iniciación del tratamiento con clozapina y otros medicamentos antipsicóticos en un estudio de las historias clínicas de 200 pacientes (158 varones, edad media en el ingreso $=$ 21,5 años, DT $=5,03$ ) con esquizofrenia de comienzo reciente $(n=152)$, trastorno esquizofreniforme $(n=12)$ o trastorno esquizoafectivo $(n=36)$, escogidos al azar a partir de 900 primeros ingresos desde 1984 a 2000 en una unidad de primera psicosis en el Centro Médico Académico de Amsterdam.

Cuatro de los 41 pacientes (el 9,8\%) con clozapina tenían TOC antes del comienzo del tratamiento. Durante éste, sus sintomas de TOC se redujeron y no se diagnosticaba ya TOC en el alta. Sin embargo, otrós cuatro pacientes (el 9,8\%) desarrollaron TOC de novo durante la terapia de clozapina.

En el grupo tratado con otros medicamentos antipsicóticos, 10 de 154 (el 6,5) mostraban TOC antes del tratamiento $\mathrm{y}$ este número se redujo a tres en el alta $(1,9 \%)$. Ningu- no de los pacientes desarrolló TOC de novo durante el tratamiento con otros medicamentos antipsicóticos. Cinco pacientes rechazaron la medicación. La terapia de clozapina se asociaba con más casos de TOC en el alta $\left(\chi^{2}\right.$ de Pearson $=6,0, \mathrm{gl}=2, P=0,05)$ y con menor reducción neta en los casos de TOC ( $\chi^{2}$ de Pearson $\left.=18,2, \mathrm{gl}=4, P=0,001\right)$ comparado con el tratamiento con otros medicamentos antipsicóticos.

Nuestros resultados indican que un subgrupo de pacientes con esquizofrenia de comienzo reciente o trastornos relacionados es susceptible de inducción de TOC durante el tratamiento con clozapina y que la clozapina se asocia con reducción del TOC en otro subgrupo. Nuestros hallazgos subrayan la complejidad de la respuesta a la clozapina en lo referido al TOC en los pacientes con trastornos esquizofrénicos. Esta complejidad de la respuesta se puede relacionar con polimorfismos del gen del receptor 5$\mathrm{HT}_{2 \mathrm{~A}}$. Estos polimorfismos se asocian con la respuesta clínica a la clozapina [1] y el TOC [4] y podrían explicar los efectos diferenciales de la clozapina en nuestros pacientes.

\section{Bibliografía}

[1] Arranz MJ, Munro J, Sham P, Kirov G, Murray RM, Collier DA, et al. Meta-analysis of studies on genetic variation in 5-HT2A receptors and clozapine response. Schizophr Res 1998;32:93-9.

[2] De Haan L, Linszen DH, Gorsira R. Clozapine and obsessions in patients with recent-onset schizophrenia and other psychotic disorder. J Clin Psychiatry 1999;60(6):364-5.

[3] Eales MJ, Layeni AO. Exacerbation of obsessive compulsive symptoms associated with clozapine. Br J Psychiatry 1994; 164:687-8.

[4] Enoch MA, Kaye WH, Rotondo A, Greenber BD, Murphy DL, Goldman D. 5-HT2A promotor polymorphism -1438G/A, anorexia nervosa, and obsessive-compulsive disorder. Lancet 1998;351:17856 [letter].

[5] Ghaemi SN, Zarate CA, Popli AP, Srinivasan SP, Pillay S, Cole JO. Is there a relationship between clozapine and obsessive-compulsive disorder? A retrospective chart review. Compr Psychiatry 1995;36:267-70.

[6] Patel B, Tandon R. Development of obsessive-compulsive symptoms dunng clozapine treatment. Am J Psychiatry 1993;150:836.

[7] Patil VJ. Development of transient obsessive-compulsive symptoms during treatment with clozapine. Am J Psychiatry 1992;149:272.

Lieuwe De Haan

Aygul Oekeneva

Therese Van Amelsvoort

Don Linszen

Clínica Adolescente, Centro Médico Académico, Departamento de Psiquiatria, Universidad, Post Box 22700, 1100 DE Amsterdam, Paises Bajos Correo electrónico l.dehaan@amc.uva.nl (L.De Haan). 